The Astronomical Journal, 130:2237-2240, 2005 November

(C) 2005. The American Astronomical Society. All rights reserved. Printed in U.S.A.

\title{
MID-INFRARED OBSERVATIONS OF THE WHITE DWARF-BROWN DWARF BINARY GD 1400
}

\author{
J. FARIHI, ${ }^{1,2}$ B. Zuckerman, ${ }^{2}$ AND E. E. BECKLIN ${ }^{2}$ \\ Received 2005 July 11; accepted 2005 July 27
}

\begin{abstract}
Fluxes are measured for the DA white dwarf plus brown dwarf pair GD 1400 with the Infrared Array Camera on the Spitzer Space Telescope. GD 1400 displays an infrared excess over the entire 3-8 $\mu$ m region consistent with the presence of a mid- to late-type L dwarf companion. A discussion is given regarding current knowledge of this unique system.
\end{abstract}

Key words: binaries: general — stars: evolution — stars: formation — stars: low-mass, brown dwarfs white dwarfs

\section{INTRODUCTION}

White dwarfs make excellent infrared targets with a favorable contrast between cool self-luminous orbiting bodies and the target star. This fact was perhaps first appreciated by Probst \& O'Connell (1982) and later by others who realized the potential for the detection of substellar companions (Probst 1983; Shipman 1986; Zuckerman \& Becklin 1987, 1992). Although low-mass stars and brown dwarfs have cool effective temperatures and are intrinsically faint, their radii $\left(R \approx 1 R_{\mathrm{J}}\right.$; Burrows et al. 1997, 2001; Chabrier \& Baraffe 2000; Chabrier et al. 2000) are approximately 10 times larger than white dwarf radii $(R \approx$ $1 R_{\oplus}$; Bergeron et al. 1995a, 1995b). Therefore, spatially unresolved low-mass stellar and substellar companions to white dwarfs can be detected in the infrared as excess emission. These facts led to the discovery of the first brown dwarf candidate, the prototype L dwarf, and the coolest known "star" for about 7 years, GD 165B (Becklin \& Zuckerman 1988). Currently, GD 165 and GD 1400 are the only two white dwarfs known to have L-type companions; these are possible and likely brown dwarfs, respectively (Kirkpatrick et al. 1999; Farihi \& Christopher 2004).

It is clear from large $(N>350)$ surveys that L dwarf companions to white dwarfs are not common, with frequency $\lesssim 0.5 \%$ (Farihi 2004; $;^{3}$ Farihi et al. 2005). While it is true that cooling brown dwarfs pass through the more luminous $\mathrm{M}$ and $\mathrm{L}$ dwarf stages faster than later stages, the lowest mass stars $\left(0.075 M_{\odot} \lesssim\right.$ $M<0.10 M_{\odot}$ ) should have spectral types in the range M6-L3 at white dwarf ages and beyond (Chabrier \& Baraffe 2000; Chabrier et al. 2000; Burrows et al. 2001). Hence, the relative dearth of both $\mathrm{L}$ and late-M dwarf companions to white dwarfs, compared to the frequency of earlier M-type companions and to the number of known L and late-M dwarfs in the field (Zuckerman \& Becklin 1992; Green et al. 2000; Wachter et al. 2003; Farihi 2004; Farihi et al. 2005), is indicative of binary star formationspecifically, low-mass companion formation to intermediate-mass stars - and not a limitation or bias in searches.

Relative to the sensitivity and wide range of separations that have been probed for L dwarfs, there is less evidence against the presence of $\mathrm{T}$ dwarf and later type companions to white dwarfs (Farihi 2004; Farihi et al. 2005; Dobbie et al. 2005). Both $\mathrm{H}_{2} \mathrm{O}$

\footnotetext{
${ }^{1}$ Gemini Observatory, Northern Operations, 670 North A'ohoku Place, Hilo, HI 96720; jfarihi@gemini.edu.

2 Department of Physics and Astronomy, University of California, 430 Portola Plaza, Los Angeles, CA 90095.

3 Available at http://www.whitedwarf.org.
}

and $\mathrm{CH}_{4}$ absorption, plus $\mathrm{H}_{2}$ collision-induced absorptions, suppress some regions of near-infrared flux in $\mathrm{T}$ dwarfs (Burgasser et al. 2002), causing them to appear blue in the $J-K$ color index. This makes $\mathrm{T}$ dwarf companions photometrically undetectable as excess emission at $2.2 \mu \mathrm{m}$, unless the white dwarf primary has $M_{K} \gtrsim 13$ mag (Farihi 2004; Farihi et al. 2005), corresponding to very cool and/or very massive degenerates $\left(T_{\text {eff }}<7000 \mathrm{~K}\right.$ for $\log g=8.0$, or $T_{\text {eff }}<9000 \mathrm{~K}$ for $\log g=8.5$; Bergeron et al. 1995a, 1995b). Although the majority of known white dwarfs do not meet these faintness criteria (McCook \& Sion 1999), ${ }^{4}$ many nearby cool degenerates have been photometrically surveyed in the near-infrared for various purposes, with no evidence of T dwarf secondaries via $K$-band excess (Bergeron et al. 1997, 2001; Leggett et al. 1998; Farihi 2004; Farihi et al. 2005). Owing to these facts, generally speaking, T-type and later companions to white dwarfs are only detectable in the near-infrared as spatially resolved objects in deep ground-based or space-based imaging, or as unresolved secondaries with high signal-to-noise ratio (S/N) spectroscopy (Burleigh et al. 2002; Farihi 2004; Farihi \& Christopher 2004; Farihi et al. 2005; Dobbie et al. 2005). For wavelengths $\gtrsim 3 \mu \mathrm{m}$, the contrast is once again favorable for the detection of the coolest brown dwarf companions (Burrows et al. 2003). However, due to large thermal background, there are very few white dwarfs bright enough to be observed from the ground at $3 \mu \mathrm{m}$ and beyond, where companions later than L-type might be detectable around a significant fraction of known degenerates. Hence, space-based imaging and spectroscopy at $\lambda \gtrsim 3 \mu \mathrm{m}$ with the Spitzer Space Telescope is currently the only way to survey a large number of white dwarfs for unresolved T-type and later brown dwarf companions.

This paper presents mid-infrared fluxes and magnitudes for GD 1400 (DA4.3+dL6.5, $d \approx 39$ pc; Farihi \& Christopher 2004; Dobbie et al. 2005) measured with the Infrared Array Camera (IRAC; Fazio et al. 2004) on Spitzer. The IRAC data are found to be consistent with expectations based on previously published ground-based near-infrared photometric and spectroscopic data.

\section{DATA AND ANALYSIS}

GD 1400 was observed with IRAC in all four channels as part of a program searching for substellar companions to nearby white dwarfs. The imaging strategy consisted of $30 \mathrm{~s}$ frame times in a 20 point, medium-scale (median move of $\sim 40^{\prime \prime}$ ) cycling dither

\footnotetext{
${ }^{4}$ Current catalog at http://www.astronomy.villanova.edu/WDCatalog/index .html
} 
TABLE 1

IRAC FLUXES FOR GD 1400

\begin{tabular}{|c|c|c|c|c|c|}
\hline Channel & $\begin{array}{c}\lambda_{0}^{\mathrm{a}} \\
(\mu \mathrm{m})\end{array}$ & $\begin{array}{c}\Delta \lambda^{\mathrm{a}} \\
(\mu \mathrm{m})\end{array}$ & $\begin{array}{c}F_{\nu} \\
(\mu \mathrm{Jy})\end{array}$ & $\mathrm{S} / \mathrm{N}$ & Magnitude \\
\hline 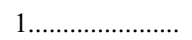 & 3.55 & 0.68 & 971 & 440 & $13.65 \pm 0.06$ \\
\hline $2 \ldots \ldots \ldots \ldots \ldots \ldots \ldots$ & 4.49 & 0.87 & 608 & 190 & $13.68 \pm 0.06$ \\
\hline 3............................ & 5.73 & 1.25 & 513 & 31 & $13.37 \pm 0.07$ \\
\hline 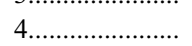 & 7.87 & 2.53 & 321 & 17 & $13.25 \pm 0.08$ \\
\hline
\end{tabular}

Notes.-Not included in the $\mathrm{S} / \mathrm{N}$ is the absolute calibration uncertainty in the IRAC instrument at the time of the pipeline processing, which was around $6 \%$ (M. Cohen 2005, private communication; Cohen 2004). This uncertainty is included in the magnitude column.

${ }^{\text {a }}$ Values taken from the IRAC Data Handbook 2.0 (Reach et al. 2005).

pattern. In this way the point-spread function is well sampled, saturation of target and candidate companions is avoided, effects of cosmic rays, detector blemishes, and bad pixels can be removed, flat-fielding errors are minimized, and detector artifacts due to bright sources on and off the chip are more easily eliminated. Thus, a total integration time of $600 \mathrm{~s}$ was achieved at each of these wavelengths: 3.6, 4.5, 5.7, and $7.9 \mu \mathrm{m}$ (Reach et al. 2005).

The individual frames were combined into a single image via the IRAC calibration pipeline, version 11.0. For single images, the basic calibrated data contain dark and sky subtraction, linearization, flat-fielding, cosmic-ray detection, flagging of bad pixels, and flux calibration. The final processed image includes pointing refinement, mosaicking (image registration onto a larger grid), masking, and co-addition with outlier rejection (Reach et al. 2005). Aperture photometry was performed on the target in the final combined image using standard IRAF tasks. Both the flux and $\mathrm{S} / \mathrm{N}$ were measured in a 2 -3 (wavelength dependent) pixel radius, with a sky annulus of $r=10-20$ pixels. This measured flux was then corrected to the standard 10 pixel aperture radius using aperture corrections found in Reach et al. (2005). The results are listed in Table 1.

\section{RESULTS AND DISCUSSION}

\subsection{2-8 $\mu \mathrm{m}$ Colors}

Little is known about the probable white dwarf plus brown dwarf spectroscopic binary, GD 1400. The cool companion was discovered, then confirmed, through photometric excess and subsequent spectroscopy in the $2.2 \mu \mathrm{m}$ region (Farihi \& Christopher 2004). To give a brief summary, its apparent lack of excess emission at $1.2 \mu \mathrm{m}$ implies that GD 1400B has a spectral type of L5.5 or later, and the lack of $\mathrm{Na}$ in its $K$-band spectrum indicates it cannot be an early $\mathrm{L}$ dwarf. Using the best available data on the white dwarf primary to assess its distance and to account for its contribution at near-infrared wavelengths, the absolute magnitude of GD 1400B would place it around spectral type L6 (Farihi \& Christopher 2004). Subsequently, an independent spectroscopic study estimated GD 1400B at spectral type L 7 through simultaneous fits of the white dwarf and brown dwarf components in an HK grism observation, with model and empirical template spectra, respectively (Dobbie et al. 2005).

The IRAC measurements of GD 1400 presented in Figure 1 and Table 1 have $\mathrm{S} / \mathrm{N}>15$ at all wavelengths. In Table 2, the expected flux from GD 1400A at IRAC wavelengths has been calculated from the data in Farihi \& Christopher (2004) and then subtracted from the total flux to produce the contribution of GD $1400 \mathrm{~B}$, with errors. It is noteworthy that using the Rayleigh-Jeans

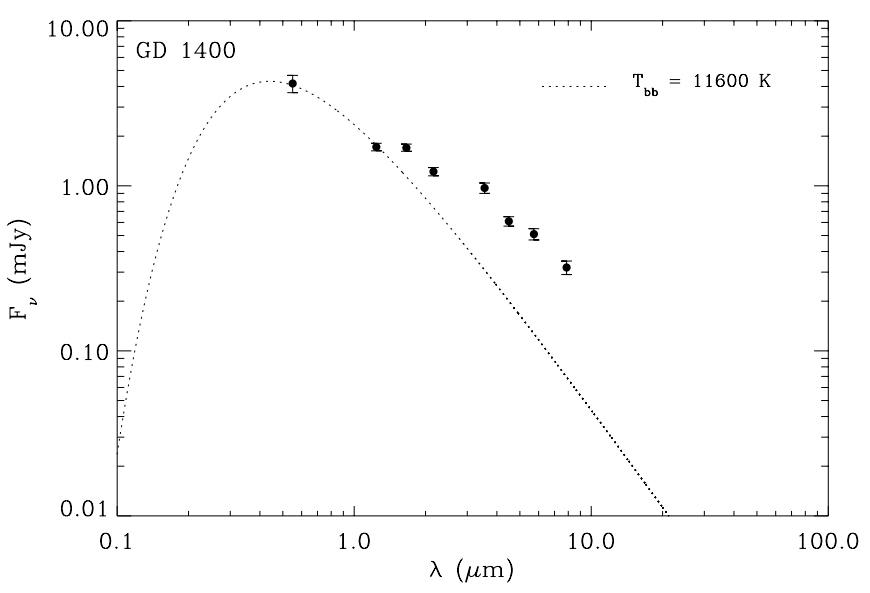

FIG. 1.-Spectral energy distribution of GD 1400, demonstrating the presence of the spatially unresolved, cool brown dwarf companion. Optical and near-infrared (VJHK) data are from Farihi \& Christopher (2004).

approximation longward of $2 \mu \mathrm{m}$ does not yield zero color, as expected for an $11,600 \mathrm{~K}$ star. This is a consequence of the IRAC zero-magnitude flux scale (Reach et al. 2005) and not a reflection of any intrinsic property of GD 1400A. For completeness, Table 2 lists the resulting deconvolved magnitudes for GD 1400B using both Rayleigh-Jeans and zero-color assumptions for GD 1400A. Table 3 lists the $2-8 \mu \mathrm{m}$ colors implied by the Table 2 magnitudes for GD 1400B, which are minimally affected by the choice of model for GD 1400A. The deconvolved magnitudes of the cool companion imply near- to mid-infrared colors consistent with those measured for isolated mid- to late- $\mathrm{L}$ dwarfs (Patten et al. 2004). The last column of Table 3 lists the ranges of $\mathrm{L}$ dwarf types consistent with each color index, based on relations in Patten et al. (2004). The 2-8 $\mu \mathrm{m}$ colors of GD 1400B are consistent with a spectral type of L5-L7, corroborating previous findings by alternate methods.

Looking at Figure 1, there appears to be a relative drop in flux at $4.5 \mu \mathrm{m}$ compared to the other three IRAC bandpass measurements. Accordingly, the [3.6]-[4.5] color index of GD 1400B is bluer than its other IRAC colors and is actually slightly negative. A slightly negative [3.6]-[4.5] color index is also seen in the majority of all L dwarfs in Patten et al. (2004) but appears to be a bit more pronounced in types L5 and later. The reason for this is probably the presence of a wide $\mathrm{CO}$ absorption feature that spans a decent portion of the $4.5 \mu \mathrm{m}$ IRAC bandpass and that becomes more pronounced at later L dwarf types (Saumon et al. 2003). Although no currently published L dwarf spectra span the entire 3-5 $\mu \mathrm{m}$ range, some ground-based $L$-band spectra of $\mathrm{L}$ dwarfs exist, and the beginnings of this $\mathrm{CO}$ feature may be what is seen to cause a drop in flux just before $4 \mu \mathrm{m}$ (Cushing et al. 2005).

The remaining IRAC data points in Figure 1 do not deviate drastically from a Rayleigh-Jeans-type slope, as seen in M dwarfs (Roellig et al. 2004; Cushing et al. 2005). Hence, it is unlikely that GD 1400B is cool enough to have formed a significant amount of $\mathrm{CH}_{4}$ yet, as L8-L9 spectral types have (Geballe et al. 2002; Cushing et al. 2005), which can cause IRAC colors to more nearly resemble those of $\mathrm{T}$ dwarfs (Patten et al. 2004).

\subsection{Origin and Evolution}

Because GD 1400AB has yet to be spatially resolved ( $a \lesssim$ 0."3; Farihi \& Christopher 2004), it remains possible that this spectroscopic binary is a radial velocity variable. It is perhaps more likely the system resides in close orbit due to the fact that 
TABLE 2

Magnitudes For GD 1400A AND B

\begin{tabular}{|c|c|c|c|c|c|}
\hline Object & $\begin{array}{c}K_{s} \\
(\mathrm{mag})\end{array}$ & $\begin{array}{c}{[3.6]} \\
\text { (mag) }\end{array}$ & $\begin{array}{c}{[4.5]} \\
(\mathrm{mag})\end{array}$ & $\begin{array}{l}{[5.7]} \\
(\mathrm{mag})\end{array}$ & $\begin{array}{c}{[7.9]} \\
(\mathrm{mag})\end{array}$ \\
\hline GD $1400 \mathrm{~A}^{\mathrm{a}}$ & $15.09 \pm 0.12$ & $15.22 \pm 0.12$ & $15.26 \pm 0.12$ & $15.30 \pm 0.12$ & $15.37 \pm 0.12$ \\
\hline GD $1400 B^{a}$. & $15.10 \pm 0.20$ & $13.94 \pm 0.10$ & $13.97 \pm 0.10$ & $13.57 \pm 0.10$ & $13.42 \pm 0.11$ \\
\hline GD $1400 A^{b}$. & $15.09 \pm 0.12$ & $15.09 \pm 0.12$ & $15.09 \pm 0.12$ & $15.09 \pm 0.12$ & $15.09 \pm 0.12$ \\
\hline GD $1400 B^{b}$ & $15.10 \pm 0.20$ & $13.98 \pm 0.10$ & $14.03 \pm 0.10$ & $13.62 \pm 0.10$ & $13.47 \pm 0.11$ \\
\hline
\end{tabular}

Noте. $-K_{s}$ data taken from Farihi \& Christopher (2004).

a Flux of GD 1400A extrapolated to IRAC wavelengths using the Rayleigh-Jeans approximation.

${ }^{b}$ Flux of GD 1400A extrapolated to IRAC wavelengths assuming zero color.

post-asymptotic giant branch (AGB) evolution predicts a bimodal distribution of orbital semimajor axes for low-mass, unevolved companions to white dwarfs (Farihi 2004). Specifically, companions close enough to orbit within the AGB envelope should spiral inward due to transfer of orbital energy into the envelope via friction (Paczyński 1976; Livio \& Soker 1984, 1988), while those outside the envelope should spiral outward due to weakened gravity from mass loss (Jeans 1924; Zuckerman \& Becklin 1987; Burleigh et al. 2002; Farihi 2004). It is not known exactly where the critical radius lies for inward versus outward orbital alteration, and it must depend on companion mass, but a reasonable assumption is on the order of a few AU based both on theory that includes tidal interactions (important for the lowest mass companions) and the fact that intermediate-mass stars should evolve to have AGB photospheres $\sim 1-2$ AU in radius (Sackmann et al. 1993; Rasio et al. 1996; Duncan \& Lissauer 1998; Siess \& Livio 1999; Pasinetti-Fracassini et al. 2001; Burleigh et al. 2002). Observations separated by 2 days in 2000 July revealed a small, $\sim 4 \%$ variation in the radial velocity of GD $1400 \mathrm{~A}$ (R. Napiwotzki 2005, private communication). The measured variation is not inconsistent with a very low mass $\left(\sim 0.06 M_{\odot}\right)$ companion with an orbital period greater than several days.

Further radial velocity monitoring of the white dwarf in the optical and/or its companion in the near-infrared, or high resolution ground- or space-based imaging, should eventually reveal the nature of the current orbital separation of the binary. Resolving the pair would be advantageous because the companion could be directly studied. On the other hand, it would be fortuitous if the system were a radial velocity variable because then the mass and radius of the secondary could be estimated. Currently, there is only a single L dwarf (binary) system with a mass measurement (Bouy et al. 2004) and no mass estimates for old brown dwarfs. There exist two independent and reliable spectroscopic fits of $T_{\mathrm{eff}}$ and $\log g$ for GD 1400A, and hence the mass of the white dwarf is fairly well constrained near $M \approx 0.7 M_{\odot}$ (Koester et al. 2001; Fontaine et al. 2003). A trigonometric parallax and

TABLE 3

2-8 $\mu \mathrm{m}$ COLORS FOR GD 1400B

\begin{tabular}{cccc}
\hline \hline Index & Color 1 & Color 2 & Sp. Type $^{\mathrm{a}}$ \\
\hline$K_{s}-[3.6] \ldots \ldots \ldots \ldots . .$. & +1.16 & +1.12 & L5-L7 \\
{$[3.6]-[4.5] \ldots \ldots \ldots .$.} & -0.03 & -0.05 & L0-L8 \\
{$[4.5]-[5.7] \ldots \ldots \ldots .$.} & +0.40 & +0.41 & L5-L8 \\
{$[5.7]-[7.9] \ldots \ldots \ldots .$.} & +0.15 & +0.15 & L0-L7 \\
\hline
\end{tabular}

Note.-Colors 1 and 2 are those implied by the magnitudes in rows 2 and 4 of Table 2, respectively.

${ }^{a}$ Range of $\mathrm{L}$ dwarf types consistent with each measured color index, based on relations in Patten et al. (2004). high-precision optical photometry would tighten up the primary mass estimate, making any secondary mass determination more reliable.

Determining the orbital parameters of this so far unique binary is critical to understanding the origin and evolution of the brown dwarf secondary. It is likely that the system formed as an extremely low mass ratio binary $\left(M_{2} / M_{1} \approx 0.02\right.$; Farihi $\&$ Christopher 2004), but it is conceivable that the companion formed in a massive disk around a $\sim 3 M_{\odot}$ main-sequence star. There have been several substellar companions detected around $\mathrm{K}$ giants (Frink et al. 2002; Mitchell et al. 2003), which are the descendents of main-sequence A and F stars. Presumably, these substellar companions formed in their respective primary progenitor disks based on their current orbital semimajor axes. Will these brown dwarfs survive the current first-ascent and ensuing asymptotic giant branches to become companion systems similar to GD 1400? Although complete evaporation or inspiral collision with the stellar core is possible inside the AGB envelope, the higher mass brown dwarfs around these $\mathrm{K}$ giants may persist, as has GD 1400B, either by eschewing the greatly expanded photosphere or simply surviving the envelope itself (Livio \& Soker 1984; Iben \& Livio 1993; Sackmann et al. 1993; Rasio et al. 1996; Duncan \& Lissauer 1998; Siess \& Livio 1999; Burleigh et al. 2002).

It is not known whether GD 1400AB shared a common envelope during the AGB phase of the primary, but hopefully more data will soon give indications one way or another. Important questions regarding this stage of evolution and its outcome are (1) Was any mass accreted by the low-mass companion?; (2) What were the initial masses and separation of the binary?; and (3) Is the pair close enough now to interact in any way that might be detectable? Ultimately, the core science is to understand the origin of the current binary stellar parameters, especially those of GD 1400B, and to discover in what ways these are products of formation, evolution, or both.

At present, there is no hard evidence that low-mass unevolved companions $\left(M \lesssim 0.3 M_{\odot}\right)$ to white dwarfs in detached, postcommon envelope binaries have accreted a significant amount of mass ( 0.01-0.1 $M_{\odot}$; Maxted et al. 1998; Chabrier \& Baraffe 2000; Farihi 2004; Farihi et al. 2005). However, there is some tentative and indirect evidence of secondary accretion from either the common envelope or the more distant AGB wind in dwarf and giant $\mathrm{K}$ stars, such as overabundances of carbon and $s$-process elements and oversized apparent radii (Pollacco \& Bell 1994; Jeffries \& Smalley 1996; Bond et al. 2003; Drake \& Sarna 2003). First, it is uncertain whether these observations imply accretion of an order that would be noticeable in the mass distribution of post-common envelope versus widely separated low-mass companions to white dwarfs; i.e., enough mass to transform a brown dwarf into a star (Farihi 2004). Second, there are a few alternative explanations for the aforementioned observations. Accretion 
from the AGB envelope or wind should take place more readily for relatively more massive ( $\mathrm{K}$ dwarf) or compact secondaries, whereas companions with oversized apparent radii can be explained by either irradiation from very hot helium-burning stars or starspots (Pollacco \& Bell 1994; O'Brien et al. 2001). It is also possible that a currently detached, post-common envelope binary was previously in a semidetached configuration, in which mass exchange might occur. This can take place if enough mass is lost adiabatically from the system to expand the orbit (Jeans 1924; Nelemans et al. 2001). There is still significant interest in this issue, and tests have been proposed to look for evidence of secondary accretion from the envelope (Sarna et al. 1995; Dhillon et al. 2002).

Another issue important for DA white dwarfs like GD 1400A is possible atmospheric pollution by winds from close, lowmass companions. In searches for metal-rich DA (DAZ) stars, white dwarfs known to have unresolved red dwarf companions are found to have $\mathrm{Ca}$ in their photospheres with significantly greater frequency $(60 \%)$ than other types of double or single white dwarf systems (6\%-20\%; Zuckerman \& Reid 1998; Zuckerman et al. 2003). Unseen companions or planetary material have often been considered a possible explanation both in particular instances and in general for the DAZ phenomenon (Holberg et al. 1997; Jura 2003; Dobbie et al. 2005). GD 1400A, observed for the SN Ia Progenitor Survey (SPY) project (Koester et al. 2001), was spectroscopically searched for $\mathrm{Ca} \mathrm{H}$ and $\mathrm{K}$ lines, which were not detected (Koester et al. 2005). Based on the published sensitivity, this should firmly rule out $\mathrm{Ca}$ abundances greater than $[\mathrm{Ca} / \mathrm{H}] \approx 9$ (see Fig. 2 of Koester et al. 2005). If the pair is close now, then there appears to be little, if any, Ca pollution of GD 1400A by any wind from GD 1400B.

\section{CONCLUSION}

The mid-infrared data from Spitzer IRAC corroborate the presence of an apparently ordinary L dwarf companion to GD 1400. In addition, the colors appear to agree with previous determinations that place the companion safely in the substellar regime, with a spectral type later than L5. So far there is no hard observational evidence to confirm or rule out a close orbit, but this scenario appears more likely based on theoretical predictions and the available data.

Ongoing and future Spitzer observations can shed some light on the frequency of ultracool low-mass companions to white dwarf stars, both as unresolved secondaries and widely separated companions (J. Farihi et al. 2006, in preparation).

This work is based on observations made with the Spitzer Space Telescope, which is operated by the Jet Propulsion Laboratory (JPL), California Institute of Technology, under NASA contract 1407. Support for this work was provided by NASA through contract 1264491 issued by JPL, Caltech.
Becklin, E., \& Zuckerman, B. 1988, Nature, 336, 656

Bergeron, P., Leggett, S., \& Ruiz, M. 2001, ApJS, 133, 413

Bergeron, P., Ruiz, M., \& Leggett, S. 1997, ApJS, 108, 339

Bergeron, P., Saumon, D., \& Wesemael, F. 1995a, ApJ, 443, 764

Bergeron, P., Wesemael, F., \& Beauchamp, A. 1995b, PASP, 107, 1047

Bond, H., Pollacco, D., \& Webbink, R. 2003, AJ, 125, 260

Bouy, H., et al. 2004, A\&A, 423, 341

Burgasser, A., et al. 2002, ApJ, 564, 421

Burleigh, M., Clarke, F., \& Hodgkin, S. 2002, MNRAS, 331, L41

Burrows, A., Hubbard, W., Lunine, J., \& Liebert, J. 2001, Rev. Mod. Phys., 73, 719

Burrows, A., Sudarsky, D., \& Lunine, J. 2003, ApJ, 596, 587

Burrows, A., et al. 1997, ApJ, 491, 856

Chabrier, G., \& Baraffe, I. 2000, ARA\&A, 38, 337

Chabrier, G., Baraffe, I., Allard, F., \& Hauschildt, P. 2000, ApJ, 542, 464

Cohen, M. 2004, in Spitzer Calibration Workshop (Pasadena: SSC), http:// ssc.spitzer.caltech.edu/ost/workshop/2004calib/pdf/cohenm.pdf

Cushing, M., Rayner, J., \& Vacca, W. 2005, ApJ, 623, 1115

Dhillon, V., Littlefair, S., Marsh, T., Sarna, M., \& Boakes, E. 2002, A\&A, 393, 611

Dobbie, P., Burleigh, M., Levan, A., Barstow, M., Napiwotzki, R., Holberg, J., Hubeny, I., \& Howell, S. 2005, MNRAS, 357, 1049

Drake, J., \& Sarna, M. 2003, ApJ, 594, L55

Duncan, M., \& Lissauer, J. 1998, Icarus, 134, 303

Farihi, J. 2004, Ph.D. thesis, UCLA

Farihi, J., Becklin, E., \& Zuckerman, B. 2005, ApJS, in press

Farihi, J., \& Christopher, M. 2004, AJ, 128, 1868

Fazio, G., et al. 2004, ApJS, 154, 10

Fontaine, G., Bergeron, P., Billères, M., \& Charpinet, S. 2003, ApJ, 591, 1184

Frink, S., Mitchell, D., Quirrenbach, A., Fischer, D., Marcy, G., \& Butler, R. 2002, ApJ, 576, 478

Geballe, T., et al. 2002, ApJ, 564, 466

Green, P., Ali, B., \& Napiwotzki, R. 2000, ApJ, 540, 992

Holberg, J., Barstow, M., \& Green, E. 1997, ApJ, 474, L127

Iben, I., \& Livio, M. 1993, PASP, 105, 1373

Jeans, J. 1924, MNRAS, 85, 2

Jeffries, R., \& Smalley, B. 1996, A\&A, 315, L19

Jura, M. 2003, ApJ, 584, L91

Kirkpatrick, J., Allard, F., Bida, T., Zuckerman, B., Becklin, E., Chabrier, G., \& Baraffe, I. 1999, ApJ, 519, 834
Koester, D., Rollenhagen, R., Napiwotzki, R., Voss, B., Christlieb, N., Domeier, D., \& Reimers, D. 2005, A\&A, 432, 1025

Koester, D., et al. 2001, A\&A, 378, 556

Leggett, S., Ruiz, M., \& Bergeron, P. 1998, ApJ, 497, 294

Livio, M., \& Soker, N. 1984, MNRAS, 208, 783 1988, ApJ, 329, 764

Maxted, P., Marsh, T., Moran, C., Dhillon, V., \& Hilditch, R. 1998, MNRAS, 300,1225

McCook, G., \& Sion, E. 1999, ApJS, 121, 1

Mitchell, D., Frink, S., Quirrenbach, A., Fischer, D., Marcy, G., \& Butler, R. 2003, AAS Meeting, 203, 17.03

Nelemans, G., Yungelson, L., Portegies Zwart, S., \& Verbunt, F. 2001, A\&A, 365,491

O’Brien, M., Bond, H., \& Sion, E. 2001, ApJ, 563, 971

Paczyński, B. 1976, in IAU Symp. 73, Structure and Evolution of Close Binary Systems, ed. P. Eggleton, S. Mitton, \& J. Whelan (Dordrecht: Reidel), 75

Pasinetti-Fracassini, L., Pastori, L., Covino, S., \& Pozzi, A. 2001, A\&A, 367, 521

Patten, B., et al. 2004, BAAS, 36, 1353

Pollacco, D., \& Bell, S. 1994, MNRAS, 267, 452

Probst, R. 1983, ApJS, 53, 335

Probst, R., \& O’Connell, R. W. 1982, ApJ, 252, L69

Rasio, F., Tout, C., Lubow, S., \& Livio, M. 1996, ApJ, 470, 1187

Reach, W. T., et al. 2005, IRAC Data Handbook, ver. 2.0 (Pasadena: SSC), http://ssc.spitzer.caltech.edu/irac/dh/iracdatahandbook2.0.pdf

Roellig, T., et al. 2004, ApJS, 154, 418

Sackmann, I., Boothroyd, A., \& Kraemer, K. 1993, ApJ, 418, 457

Sarna, M., Dhillon, V., Marsh, T., \& Marks, P. 1995, MNRAS, 272, L41

Saumon, D., Marley, M., \& Lodders, K. 2003, preprint (astro-ph/0310805)

Shipman, H. 1986, in Astrophysics of Brown Dwarfs, ed. M. C. Kafatos, R. S. Harrington, \& S. P. Maran (New York: Cambridge Univ. Press), 71

Siess, L., \& Livio, M. 1999, MNRAS, 304, 925

Wachter, S., Hoard, D., Hansen, K., Wilcox, R., Taylor, H., \& Finkelstein, S. 2003, ApJ, 586, 1356

Zuckerman, B., \& Becklin, E. 1987, ApJ, 319, L99

. 1992, ApJ, 386, 260

Zuckerman, B., Koester, D., Reid, I., \& Hünsch, M. 2003, ApJ, 596, 477

Zuckerman, B., \& Reid, I. 1998, ApJ, 505, L143 